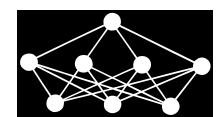

\title{
A NEW ARTIFICIAL INTELLIGENCE OPTIMIZATION METHOD FOR PCA BASED UNSUPERVISED CHANGE DETECTION OF REMOTE SENSING IMAGE DATA
}

\author{
U.H. Atasever, M.H. Kesikoglu, C. Ozkan*
}

\begin{abstract}
In this study, a new artificial intelligence optimization algorithm, Differential Search (DS), was proposed for Principal Component Analysis (PCA) based unsupervised change detection method for optic and SAR image data. The model firstly computes an eigenvector space using previously created $k \times k$ blocks. The change detection map is generated by clustering the feature vector as two clusters which are changed and unchanged using Differential Search Algorithm. For clustering, a cost function is used based on minimization of Euclidean distance between cluster centers and pixels. Experimental results of optic and SAR images proved that proposed approach is effective for unsupervised change detection of remote sensing image data.
\end{abstract}

Key words: unsupervised change detection, differential search, PCA

Received: May 26, 2014

DOI: $10.14311 /$ NNW.2016.26.008

Revised and accepted: March 10, 2016

\section{Introduction}

One of the most important subjects of remote sensing discipline is to examine the temporal variation of a specific zone. The existing differences in land cover or land use can be identified by using satellite images of different periods. Change detection approaches can effectively be used for solving important problems such as observing forest and agricultural areas, identifying the changes in land cover and identifying the spatial difference after a forest fire $[7,10,12,14,20]$. Change detection is separated into two groups as supervised and unsupervised. But in solving real world problems, unsupervised approach is more preferable than supervised approach. The reason lying under preference is that to provide the training data to be used in supervised approach is difficult while unsupervised approach does not need a training data [17]. Although there are a lot of unsupervised change detection methods for both optic and SAR remote sensing images, pre-processing steps consisting of geometric and radiometric corrections are generally needed $[10$, $17]$.

${ }^{*}$ Umit Haluk Atasever, Mustafa Hayri Kesikoglu - Corresponding author, Coskun Ozkan, Department of Geomatics Engineering, Erciyes University, 38039, Kayseri, Turkey, uhatasever@erciyes.edu.tr, hayrikesikoglu@erciyes.edu.tr, cozkan@erciyes.edu.tr 
Change vector analysis (CVA), image differencing, principal component analysis (PCA), image rationing are the basic approaches used in change detection process $[4,6,18]$. Among them, image differencing is the most commonly used approach.

Many unsupervised change detection methods have been improved over the last years. Some of them are EM+MRF based [2, 24], PCA and K-Means based [5], Fuzzy Local Information C-Means Clustering Algorithm (FLICM) [11], Reformulated FLICM (RLICM) [11], Hard C-Means (HCM), Hard C-Means hybridized with Simulated Annealing (SA-HCM), Hard C-Means Hybridized with Genetic Algorithm (GA-HCM), Fuzzy C-Means (FCM) Fuzzy C-Means Hybridized with Genetic Algorithm (GA-FCM) [9]. HCM, SA-HCM, GA-HCM and GA-FCM are generally used for optic images, FLICM and RFLICM are used for SAR images and PCA K-Means, FCM and EM+MRF can be used for both optic and radar images $[2,5,9-11,24]$.

After pre-processing, some of the change detection methods need to generate the feature space from the images of different dates by the aid of image differencing or image rationing. And then this feature space is clustered into two clusters as changed and unchanged [9, 16, 21]. One of the most important components increasing the success of the operation is the performance of the clustering algorithm. A number of algorithms have been used with this purpose in many studies $[3,5,9,11]$. Over the last decade, artificial intelligence (AI) optimization algorithms have been tested for unsupervised classification, i.e. clustering. The studies show that these algorithms have the potential to outperform the classical clustering techniques $[1,15,25]$. Although there are a number of AI optimization algorithms such as Partial Swarm Optimization (PSO), Genetic Algorithm (GA) and Artificial Bee Colony (ABC) used for clustering, as a new AI optimization algorithm, Differential Search Algorithm (DS) has not been tested for clustering purpose in change detection so far. DS algorithm outperformed many conventional AI algorithms in many standard multimodal tests [8]. It is a very powerful algorithm with fast convergence ability.

In this study, Differential Search Algorithm (DS) was used as a clustering method in an automatic PCA based unsupervised change detection approach developed by Celik (2009) [5]. This change detection approach is automatic, fast and can be used in both optical and SAR remote sensing images. Its clustering phase is the most arguable and developable step because of the fact that resulting change and unchanged regions are categorized by clustering process and many different methods exist. In this approach, after a difference image is obtained from multi-temporal images, the difference image is separated into $k \times k$ non-overlapping blocks and transformed into a feature space by PCA. Then, a min-max normalization is applied to the feature space and the obtained data set is clustered into two groups with Differential Search Algorithm (DS) as changed and unchanged areas. According to this, the relationship between PCA and DS algorithm is that PCA constructs features and DS transforms these features into clusters. Finally, performance tests are done through ground truth maps of optic and SAR image data sets. 


\section{PCA Based Unsupervised Change Detection with DS (PCA-DS Approach)}

In the first step of the method, two multi-temporal images must be registered to each other. If it is necessary, the radiometric distortions coming from sensor, atmosphere, etc. must be corrected at this step. Then, second step is the calculation of the difference image. Let $X_{\text {time } 1}$ and $X_{\text {time2 }}$ be the images belonging to same geographical areas in different dates and $X_{\text {diff }}$ be difference image. For optical images, $X_{\text {diff }}$ is calculated by subtracting the image density values of $X_{\text {time }}$ from $X_{\text {time2 }}$ and then absolute value of $X_{\text {diff }}$ is calculated $[2,5]$. The equation for optical images is defined as

$$
X_{\text {diff }}=\left|X_{\text {time2 }}-X_{\text {time1 }}\right| \text {. }
$$

For SAR images, $X_{\text {diff }}$ is calculated by subtracting the logarithmic values of $X_{\text {time } 2}$ and $X_{\text {time1 }}$ then absolute value of $X_{\text {diff }}$ is calculated. The equation for SAR images is defined as [3]

$$
X_{\text {diff }}=\left|\log \frac{X_{\text {time2 }}}{X_{\text {time } 1}}\right|=\left|\log \left(X_{\text {time2 }}\right)-\log \left(X_{\text {time } 1}\right)\right|,
$$

where log is natural logarithm.

In the third step, the difference image $X_{\text {diff }}$ is separated into $k \times k$ blocks in a lexicographical order. Let this obtained matrix be $\mathbf{M}_{\text {diff. }}^{p}$. Eigenvectors of the $\mathbf{M}_{\text {diff }}^{p}$ matrix are generated with PCA. In $\mathbf{M}_{\text {diff }}^{p}, p$ is an index value with

$$
1 \leq p \leq S\lfloor(R \times C) /(k \times k)\rfloor,
$$

where $S$ is the number of column vector sets in $\mathbf{M}_{\text {diff }}^{p}, R$ is the number of rows of $X_{\text {diff }}, C$ is the number of columns of $X_{\text {diff }}$. Since it has shown that $k=3$ generates more successful results [5], $k$ value is set as 3 in this study. A mean vector $(\boldsymbol{\Phi})$ is calculated from $\mathbf{M}_{\text {diff }}^{p}$ along the columns as

$$
\mathbf{\Phi}=\frac{1}{\mathbf{S}} \sum_{p=1}^{\mathbf{S}} \mathbf{M}_{\mathrm{diff}}^{p}
$$

After that, this mean vector is subtracted from each column vector of $\mathbf{M}_{\text {diff }}^{p}$ matrix

$$
\boldsymbol{\Delta}_{p}=\mathbf{M}_{\mathrm{diff}}^{p}-\boldsymbol{\Phi}
$$

where $\boldsymbol{\Delta}_{p}$ difference vector set. Then, covariance matrix $(\mathbf{C M})$ is calculated with matrix algebra as

$$
\mathbf{C M}=\frac{1}{\mathbf{S}} \sum_{p=1}^{\mathbf{S}} \boldsymbol{\Delta}_{p}\left(\boldsymbol{\Delta}_{p}\right)^{\mathbf{T}}
$$

where ()$^{T}$ is transposing process. Eigenvalues $\left(\lambda_{i}\right)$ and corresponding eigenvectors $\left(e_{i}\right)$ of the covariance matrix $(\mathbf{C M})$ are computed. Then the related eigenvectors for reducing of effective dimension are selected by Cumulative Percent Variance 
(CVP) selection criteria which are subjective but highly effective selection criteria for real data sets [23] as follows:

$$
\operatorname{CVP}(n)=100\left(\frac{\sum_{i=1}^{n} \lambda_{i}}{\sum_{i=1}^{k \times k} \lambda_{i}}\right) .
$$

In CVP, the eigenvectors are sorted in descending order in terms of eigenvalues and the first bundle of eigenvectors that provides a reasonable percent (e.g. 90\%, $95 \%$ or $99 \%$ ) is selected which reduces the effective dimension. These selected eigenvectors are used to project pixel values in $\mathbf{M}_{\text {diff }}(x, y)$ into feature vector space as

$$
v(x, y)=\left[\begin{array}{c}
v 1 \\
v 2 \\
v 3 \\
\vdots \\
v_{\sigma}
\end{array}\right]
$$

where $1 \leq n \leq k^{2}, v_{\sigma}=\left(e_{i}\right)^{T}\left(\mathbf{M}_{\mathrm{diff}}(x, y)-\Phi\right), 1 \leq i \leq n, 1 \leq x \leq R, 1 \leq y \leq C$ and $n$ is the number of eigenvectors used for projecting $\mathbf{M}_{\text {diff }}(x, y)$ onto eigenvector space.

The later step is the normalization of the feature vector space $v(x, y)$ into $[0,1]$ interval in order to boost the performance of DS optimization. This technique, called also min-max normalization, is performed according to the following equation:

$$
\varphi_{\text {new }}=\frac{\varphi-\varphi_{\min }}{\varphi_{\max }-\varphi_{\min }} .
$$

In Eq. (9), $\varphi_{\text {new }}$ is the normalized value; $\varphi, \varphi_{\min }$ and $\varphi_{\max }$ are the original value, minimum value and maximum value in the feature space, respectively. The tests of this study show that this type of normalization increases the effectiveness of DS in terms of convergence speed.

In the last step, feature vector space which min-max normalization has been done is clustered into two classes by using DS algorithm. As in all of the optimization methods, DS algorithm also needs an objective function for minimization. The objective function used in this study is given as

$$
\begin{gathered}
c_{j}=\left\{x_{i} ; \min \left(\left\|x_{i}-\operatorname{cnt}_{j}\right\|_{2}\right)\right\}, \\
\underset{\operatorname{cnt}_{j}}{\arg \min } \sum\left\|c_{j}-\operatorname{cnt}_{j}\right\|_{2} .
\end{gathered}
$$

In Eqs. (10) and (11), \|\|$_{2}$ is Euclidean distance, $x_{i}$ is $i$-th pixel, cnt $_{j}$ is the center of the $j$-th class, $c_{j}$ represents all of the pixels in the $j$-th class. As can be seen in the objective function, DS optimizes the cluster centers so that these centers provide the least minimum total distance between the cluster centers and the pixels of each cluster in each iteration.

After the clustering, change detection map (CDM) is ready. Since DS algorithm initializes the cluster centers randomly, the cluster labels of change and unchanged regions can vary from simulation to simulation, i.e. the change cluster doesn't 
always obtain the same label such as 1 or 2 . In order to solve this problem, a simple label correction process can be done as

$$
C D M=\left\{\begin{array}{ll}
C D M & m_{1}=2 \text { and } m_{2}=1 \\
(C D M \times(-1))+3, & m_{1}=1 \text { and } m_{2}=2
\end{array}\right\},
$$

where $m_{1}$ and $m_{2}$ are label values of pixels in changed and unchanged areas, respectively.

One of the most important characteristics of the proposed approach is a PCA block based approach: Firstly, the difference image prepared and then pixel blocks with size of $k \times k$ are used instead of individual pixels. These blocks are transformed into a new feature space by PCA. By this way, local consistency can be improved upon classical approaches. Another prominent feature of the approach is that a new evolutionary optimization DS algorithm is used for unsupervised classification instead of Fuzzy C-means or K-Means. Thus, the potential of DS algorithm in the change detection applications of remotely sensed images can be revealed.

\section{Differential Search (DS)}

DS is a population-based iterative meta-heuristic optimization algorithm. It was developed by Civicioglu to solve optimization problems having real values [8]. DS algorithm simulates Brownian-like random walk action used by a superorganism with the purpose of migrating. As the fertility of food areas changes depending on climate, a lot of species migrate to more fertile fields. A lot of species such as birds, fire ants and bees have seasonal migration cycle. Migrating species generate superorganisms involving a number of individuals. After that, superorganisms start to change the location towards more productive areas. The movement of the superorganisms can be explained by Brownian-like random walk [8].

Many of predatory species control the fertility of that habitat before migrating to a new habitat. If the potential of this habitat is enough for the needs of ten super organisms, it settles in this new area temporarily and goes on migration with goal of finding more productive regions. The population consisting of candidate solutions in DS algorithm means a migrating superorganism. This superorganism selects the good locations as stop-over site in habitat. Then the superorganism clans that find a good accommodation area migrate there.

The number of artificial organisms (clans) $\left(i, e ., X_{i}, i=\{1,2,3, \ldots, N\}\right)$ forming superorganism (i.e., Superorganism $g, g=1,2,3, \ldots$, maxgen) is equal to the dimension related optimization problem. In DS algorithm a member of superorganism's initial position is calculated by using following equation:

$$
x_{i, j}=\operatorname{rand}\left(\operatorname{up}_{j} \lim _{j}-\operatorname{low} \lim _{j}\right)+\operatorname{low} \lim _{j},
$$

where $\mathrm{N}$, maxgen, $\operatorname{uplim}_{j}$ and $\operatorname{lowlim}_{j}$ values are population number, generation number and habitat boundaries defined for $j$-th elements of clans, respectively [8].

In DS algorithm, stopover site search process can be explained with a simple Brownian-like random walk model [22]. Randomly selected individuals from the clan in limited number move on from important parameters to their donor $=$ 
$\left[X_{\text {Rand-Shuffling }(i)}\right]$ targets for the success of migration process so as to find stopover sites. Rand-Shuffling function is used for changing the placement of the components in $X_{i}$ set. The changes in the locations of artificial organisms are identified by using scale factor. The scale factor is obtained by gamma random number generator which is controlled by uniform number generator. The operating range of this number generator is $[0,1]$. Besides that, artificial superorganism can move in many different directions with this kind of tip number generator. A new stop-over site in DS is calculated as [8]:

$$
\text { StopoverSite }=(\text { SOrganism }+ \text { SFactor }) \times(\text { Donor }- \text { SOrganism }),
$$

where SOrganism is a superorganism and SFactor is a scale factor.

In Differential Search Algorithm, three different stop-over site producing mechanisms are defined as Bijective DS, Surjective DS and Elitist DS. In Bijective DS, every clan searches its habitat by going to a different clan. Bijective DS is suitable for using multi-modal problems. In Surjective DS, a clan has the tendency of going another clan existing in a more productive location. It can be preferred for partly multimodal and mostly unimodal problems. Elitist DS makes all the clans go to the clan presenting in the most productive area. It is a quite fast mechanism and can be used especially for unimodal problems. In addition to this, there are different defined random number generators which can be used for generating Scale Factor. In this study, Standard normal distributed random number generator was preferred. Which individuals in artificial organism generating superorganism will join the searching is determined at the end of a random process. The parameters of DS algorithm are $p_{1}, p_{2}$, stop over site production mechanism and scale factor. These parameters effect the convergence speed of DS for calculating the class centers. $p_{1}$ and $p_{2}$ control the mutation variable: $p_{1}$ is used to determine the type of mutation strategy randomly and $p_{2}$ defines the variation for the mutation strategy. The experimental tests confirm that the optimum value for $p_{1}$ and $p_{2}$ was $0.3 \times$ rand [8].

Stop over site production mechanism determines the search direction of DS algorithm: Elitist DS focuses on the best solution in each generation, Bijective DS searches randomly all over the solution space; Surjective DS partially focuses on the best solution. As given in Experimental study-II section, Bijective DS gives the best results for all datasets.

Scale factor is a parameter which is updated according to the type of the random number generator in each generator and aims to accelerate the convergence speed by searching solution space by multiplying with mutation variable. Although there are 6 different scale factor production mechanisms, the tests show that function of $R=1 /$ normrnd $(0,5)$ gives the best results for all data sets.

\section{Datasets, Experimental Results and Discussions}

In this paper, both SAR (Bern and Ottawa dataset) and optic (Sardinia and Mexico dataset) images are used. All datasets were downloaded from Pudn website (Accessed 12 September 2013). In order to remove the speckle noise, SAR images were filtered by the Enhanced Lee Filter $(5 \times 5$ windows $)$. The fourth band of 
Atasever U.H., Kesikoglu M.H., Ozkan C.: A new artificial intelligence...

the multispectral images was selected for change detection process because of the reflectance characteristics of water, land and vegetation objects [13]. Moreover, a ground truth map was employed to calculate the performance $[3-5,10,21]$. The measures below were used for quantitative analysis of developed approach:

- False Alarm (FA): Number of unchanged pixels identified as changed pixels.

- Missed Alarm (MA): Number of changed pixels identified as unchanged pixels.

- Total Error (TE): Total number of incorrect categorized, which is sum of FA and MA.

- Total Error Rate (TER): Ratio of sum of FA and MA to number of image pixels $(\mathrm{PN}) .(\mathrm{TER}=(\mathrm{FA}+\mathrm{MA}) / \mathrm{PN} \times 100)$

\section{Experimental Study-I}

In this section, Sardinia (Landsat 5 TM) dataset and Bern dataset were used. Sardinia image set belongs to Mulargia Lake in Sardinia Island from 1995 and 1996 years. In Sardinia data, the water level of Mulargia Lake increased between two dates and its nearby sites were flooded. The image sizes are $300 \times 412$ pixels. Because the histograms of multi-temporal Landsat 5 images are very similar to each other, any radiometric correction was not applied. Sardinia dataset and ground truth image are shown in Fig. 1. The SAR image set is a C-band ERS-2 dataset of Bern, Switzerland obtained by European Remote Sensing (ERS)-2 satellite in April and May in 1999. In Bern data, The Aare River overflowed and submerged the partial part of Bern city. The image sizes are $301 \times 301$ pixels. The filtered Bern dataset and ground truth change detection map is shown in Fig. 2.

In the application of Sardinia Landsat 5 image data, PCA-DS approach was compared with HCM, EM+MRF, PCA-KMeans, FCM, SA-HCM, G-HCM and GFCM methods. Except PCA-K Means, results of the other methods were taken from the original papers cited in Tab. I. Thus, the efficiency of the PCA-DS approach was analyzed for optic images. The visual results and error values obtained from ground truth map data are given in Fig. 3 and Tab. I, respectively. As seen from Tab. I, PCA-DS approach yielded the best change detection accuracy in all

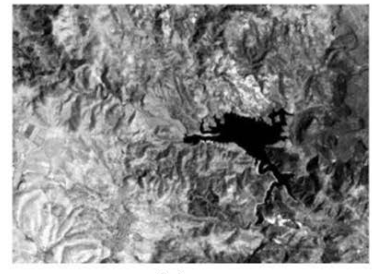

(a)

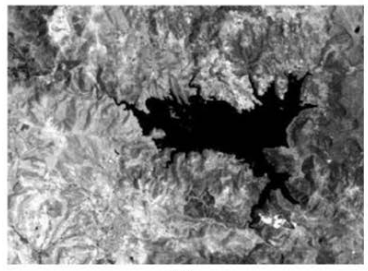

(b)

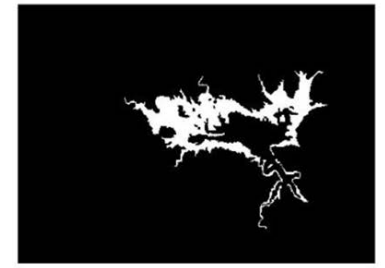

(c)

Fig. 1 Multitemporal Landsat 5 TM images of Mulargia (Band 4): (a) September 1995, (b) September 1996, (c) Ground truth. 


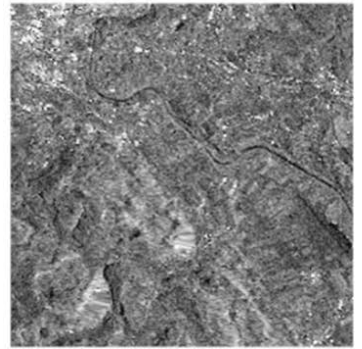

(a)

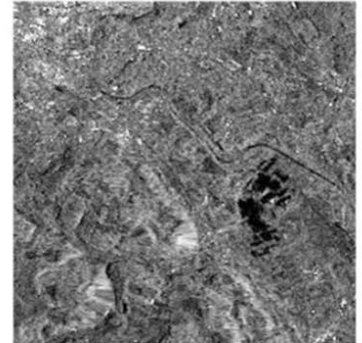

(b)

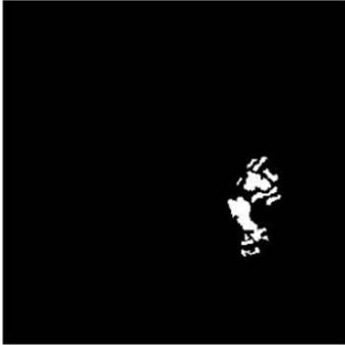

(c)

Fig. 2 Multitemporal SAR Images of Bern: (a) April 1999, (b) May 1999, (c) Ground truth.

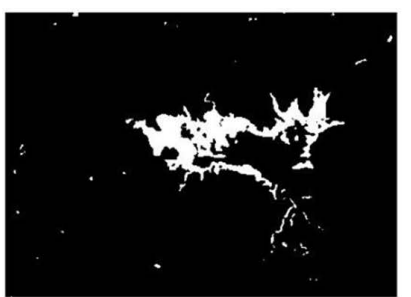

(a)

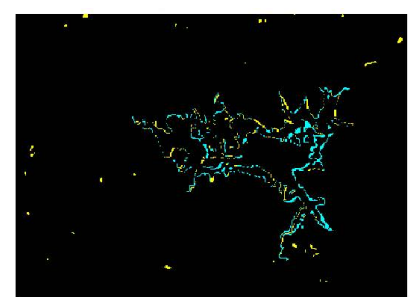

(b)

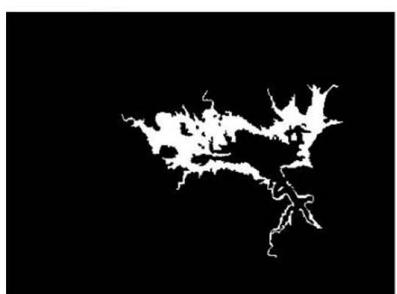

(c)

$$
\begin{aligned}
& \square \text { False Alarm } \\
& \square \text { Missed Alarm }
\end{aligned}
$$

Fig. 3 Change detection result of Sardinia data set, (a) PCA-DS approach, (b)Error Map (False and Missed Alarms) of PCA-DS, (c) Ground truth.

\begin{tabular}{lllll}
\hline Change Detection Method & $\begin{array}{l}\text { False } \\
\text { Alarm }\end{array}$ & $\begin{array}{l}\text { Missed } \\
\text { Alarm }\end{array}$ & $\begin{array}{l}\text { Total } \\
\text { Error }\end{array}$ & $\begin{array}{l}\text { Total } \\
\text { Error } \\
\text { Rate }\end{array}$ \\
\hline HCM [9] & 275 & 4133 & 4408 & 3.56 \\
EM+MRF $(\beta=1.7)[19]$ & 3856 & 289 & 4145 & 3.35 \\
SA-HCM [9] & 425 & 2727 & 3152 & 2.55 \\
G-HCM [9] & 3006 & 132 & 3138 & 2.53 \\
FCM [9] & 494 & 2246 & 2740 & 2.21 \\
G-FCM [9] & 627 & 1983 & 2610 & 2.11 \\
PCA-Kmeans Based [5] & 1603 & 832 & 2435 & 1.97 \\
Proposed Approach & $\mathbf{7 4 4}$ & $\mathbf{1 4 0 5}$ & $\mathbf{2 1 5 9}$ & $\mathbf{1 . 7 3}$ \\
\hline
\end{tabular}

Tab. I The quantitative results of Sardinia Landsat 5 TM image dataset.

methods. These results were obtained from DS with Bijective stop-over site production mechanism. In this mechanism, the scale factor is an inverse random number coming from a normal distribution with 0 mean and standard deviation of 5 . 
Population and generation number values were 10 and 500, respectively. These parameters were also used for SAR application. However, PCA-DS approach yielded better results than other methods for Sardinia dataset.

In the application of ERS-2 SAR image data of Bern, PCA-DS approach was compared with PCA-KMeans, FCM, FLICM and RFLICM methods. Except PCAKMeans, the results of other the methods were taken from the original papers cited in Tab. II. Quantitative change detection results are listed in Tab. II. As seen from Fig. 4 and Tab. II, PCA-DS approach could generate quite successful results, i.e. except RFLICM method, PCA-DS outperforms other change detection methods for SAR data.

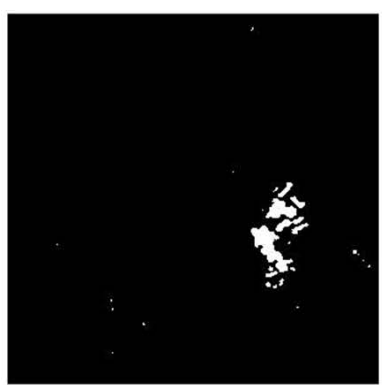

(a)

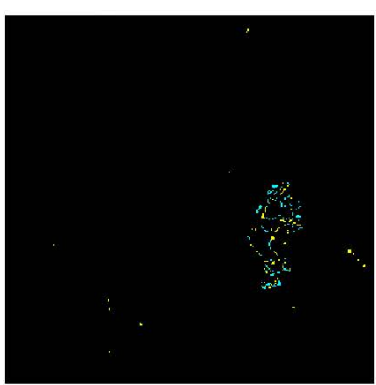

(b)

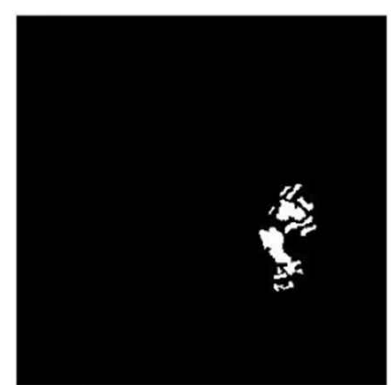

(c)

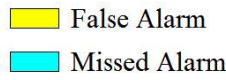

Fig. 4 Change detection result of Bern data set. (a) Proposed approach, b) Error Map (False and Missed Alarms) of PCA-DS, (c) Ground truth.

\begin{tabular}{lllll}
\hline Change Detection Method & $\begin{array}{l}\text { False } \\
\text { Alarm }\end{array}$ & $\begin{array}{l}\text { Missed } \\
\text { Alarm }\end{array}$ & $\begin{array}{l}\text { Total } \\
\text { Error }\end{array}$ & $\begin{array}{l}\text { Total } \\
\text { Error } \\
\text { Rate }\end{array}$ \\
\hline FCM [11] & 507 & 61 & 568 & 0.626 \\
FLICM [11] & 137 & 169 & 306 & 0.337 \\
PCA-Kmeans Based [5] & 146 & 158 & 304 & 0.335 \\
RFLICM [11] & 133 & 159 & 292 & 0.322 \\
Proposed Approach & $\mathbf{1 6 0}$ & $\mathbf{1 3 3}$ & $\mathbf{2 9 3}$ & $\mathbf{0 . 3 2 3}$ \\
\hline
\end{tabular}

Tab. II The quantitative change detection results of Bern dataset.

\section{Experimental Study-II}

In this section, Mexico and Ottawa image set was used for experimental studies. Also proposed approach was examined in point of DS parameters in this experimental study. The Ottawa image set is two SAR images (290x350 pixels) over 
Ottawa acquired by the Radarsat SAR sensor at Ottawa, Canada in July 1997 and August 1997. Ottawa data consists of before and after images of flood occurred in 1997 by Red River. The Mexico data set belongs to an area in Mexico acquired by Landsat $7 \mathrm{ETM}+$ from April 2000 and 2002. In Mexico data, the fire occurred between two dates destroyed the vegetation in a wide region and its environmental effect was imaged in 2002.

In the applications of Ottawa and Mexico image sets, PCA-DS approach was compared with PCA-KMeans which can generate good results for both optic and SAR images. Quantitative change detection results and computation times are listed in Tab. III. As seen from Tab. III and Fig. 5 and 6, proposed approach yielded better results for Ottawa and Mexico image sets. From a computational burden point of view, PCA-DS approach is more complex and certainly slower PCA-Kmeans method because of clustering phase takes longer for all applications since DS is not a built-in function as Kmeans in Matlab as seen in Tab. III.

\begin{tabular}{ccccccc}
\hline & $\begin{array}{c}\text { Change Detection } \\
\text { Method }\end{array}$ & $\begin{array}{c}\text { False } \\
\text { Alarm }\end{array}$ & $\begin{array}{c}\text { Missed } \\
\text { Alarm }\end{array}$ & $\begin{array}{c}\text { Total } \\
\text { Error }\end{array}$ & $\begin{array}{c}\text { Total } \\
\text { Error Rate }\end{array}$ & $\begin{array}{c}\text { Comp. } \\
\text { Time }[\mathrm{s}]\end{array}$ \\
\hline Ottawa & PCA-Kmeans [5] & 583 & 1901 & 2484 & 0.024 & 0.7 \\
Image set & PCA-DS & $\mathbf{8 5 6}$ & $\mathbf{1 5 7 4}$ & $\mathbf{2 4 3 0}$ & $\mathbf{0 . 0 2 3}$ & $\mathbf{2 6 . 6 8}$ \\
Mexico & PCA-Kmeans [5] & 1024 & 3662 & 4686 & 0.018 & 1.38 \\
Image set & PCA-DS & $\mathbf{1 5 8 9}$ & $\mathbf{2 4 2 8}$ & $\mathbf{4 0 1 7}$ & $\mathbf{0 . 0 1 5}$ & $\mathbf{7 4 . 7 6}$ \\
\hline
\end{tabular}

Tab. III The quantitative results of Ottawa and Mexico dataset.

Because of Differential Search Algorithm's and dataset structure; accuracy can vary depending on stop-over site mechanism and scale factor. To examine effectiveness of stop-over site producing mechanism and scale factor, PCA-DS approach was run with Ottawa image set with each mechanism and scale factor and best results are shown in Tab. IV and Tab. V (Population and generation number values are 10 and 200, respectively.). In this study, the best accuracy for all datasets was obtained by Bijective DS for stop-over site mechanism and $R=1 /$ normrand $(0,5)$ for scale factor. Tab. V illustrates effect of different scale factors: $R=\operatorname{lognrnd}($ rand, $5 \times$ rand) returns an array of random numbers generated from the lognormal distribution with rand mean and standard deviation of

\begin{tabular}{lcc}
\hline $\begin{array}{l}\text { Stop-over Site } \\
\text { Producing Mechanism }\end{array}$ & $\begin{array}{c}\text { Total } \\
\text { Error }\end{array}$ & $\begin{array}{c}\text { Total } \\
\text { Error Rate }\end{array}$ \\
\hline Surjective & 3339 & 0.033 \\
Elitist & 3274 & 0.032 \\
Bijective & $\mathbf{2 4 3 0}$ & $\mathbf{0 . 0 2 3}$ \\
\hline
\end{tabular}

Tab. IV The quantitative results of Ottawa dataset in point of stop-over site producing mechanism. 
Atasever U.H., Kesikoglu M.H., Ozkan C.: A new artificial intelligence...

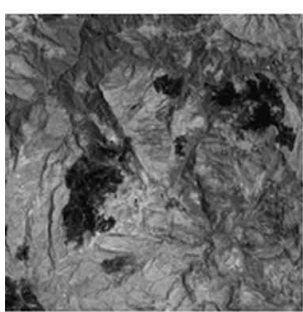

(a)

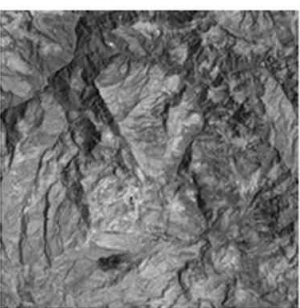

(b)

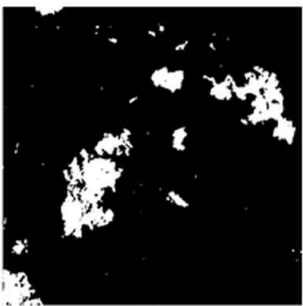

(e)

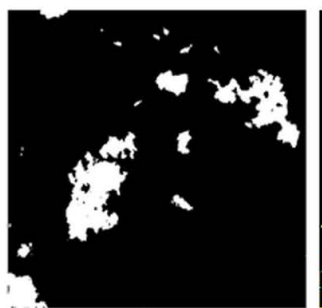

(c)

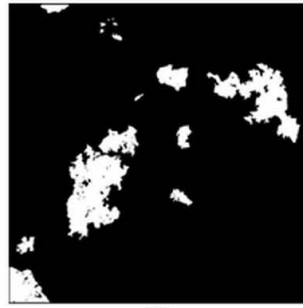

(f)

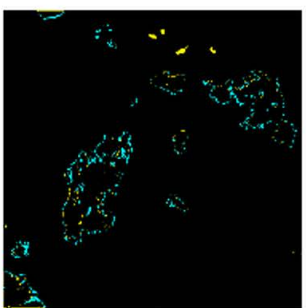

(d)

$\square$ False Alarm

$\square$ Missed Alarm

Fig. 5 Multitemporal Radarsat images of Mexico: (a) April 2000, (b)May 2002, (c) Result of PCA-DS Approach, (d) Error Map (False and Missed Alarms) of PCA-DS (e) Result of PCA-Kmeans Approach, (f) Ground Truth.

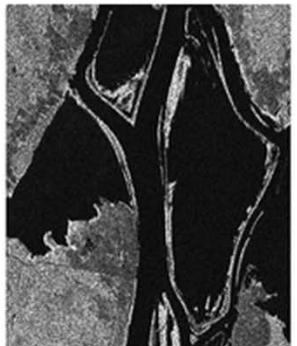

(a)

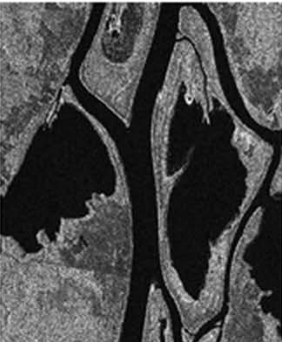

(b)

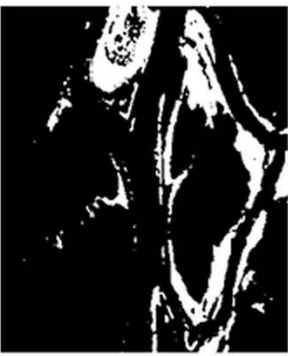

(e)

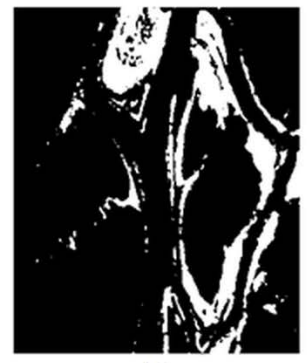

(c)

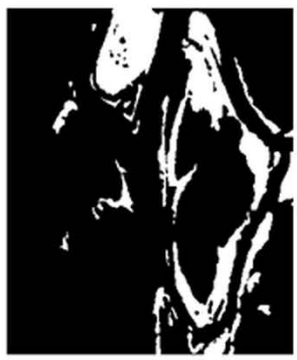

(f)

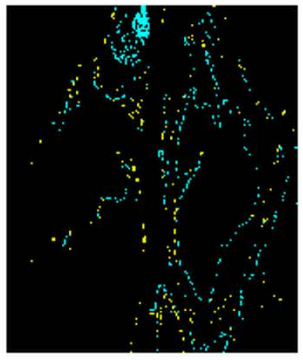

(d)

Fig. 6 Multitemporal Radarsat images of Ottawa: (a) July 1997, (b) August 1997, (c) Result of PCA-DS Approach,(d) Error Map(False and Missed Alarms) of PCA$D S$ (e) Result of PCA-Kmeans Approach, (f) Ground Truth. 


\begin{tabular}{lcc}
\hline Scale factor & Total Error & Total Error Rate \\
\hline$R=$ lognrnd(rand, $5 \times$ rand) & 4424 & 0.043 \\
$R=1 /$ gamrnd $(1,0.5)$ & 3893 & 0.038 \\
$R=1 /$ normrnd $(0.5,0.5)$ & 3675 & 0.036 \\
$R=4 \times$ randg & 3225 & 0.031 \\
$R=4 \times$ randn & 2737 & 0.027 \\
$R=1 /$ normrnd $(0,5)$ & $\mathbf{2 4 3 0}$ & $\mathbf{0 . 0 2 3}$ \\
\hline
\end{tabular}

Tab. V The quantitative results of Ottawa dataset in point of scale factor.

$5 \times$ rand; $R=$ gamrnd $(1,0.5)$ generates random numbers from the gamma distribution with shape parameters in 1 and scale parameters in 0.5 ; randg returns a scalar random value chosen from a gamma distribution with unit scale and shape; randn returns a pseudorandom scalar drawn from the standard normal distribution; rand returns a pseudorandom scalar drawn from the standard uniform distribution on the open interval $(0,1)[8]$.

\section{Conclusions}

The clustering, i.e. unsupervised classification, has an important role in change detection analysis of remotely sensed images as well as computer vision applications. Since any priori thematic information does not exist, different approaches in clustering produce different clusters. So, in this study a new artificial intelligence optimization method, Differential Search, was proposed for clustering in change detection analysis of both optic and SAR remote sensing images. DS algorithm was blended with an automatic PCA based unsupervised change detection method, i.e. PCA-DS approach. The performance of PCA-DS approach was compared with a number of change detection techniques. The applications with Sardinia, Bern, Ottawa and Mexico data sets showed that the blending with DS based clustering made the approach more effective and successful. Also proposed approach was examined in point of stop-over site production mechanism and scale factors. The optimum values of DS parameters for both optical and Radar data sets were determined and reported in the study. DS relatively needs less parameters and it is a robust algorithm. With the help of quantitative results, it can be concluded that DS algorithm had a very promising potential as a clustering method in PCA based change detection.

\section{Acknowledgement}

This work was supported by Scientific Research Projects Department of Erciyes University [FBA-2013-4643]. 


\section{Atasever U.H., Kesikoglu M.H., Ozkan C.: A new artificial intelligence...}

\section{References}

[1] ATASEVER U.H., CIVICIOGLU P., BESDOK E., OZKAN C. A New Unsupervised Change Detection Approach Based On DWT Image Fusion And Backtracking Search Optimization Algorithm For Optical Remote Sensing Data. Int. Arch. Photogramm. Remote Sens. Spatial Inf. Sci. 2014, XL-7, pp. 15-18. doi: 10.5194/isprsarchives-XL-7-15-2014.

[2] BRUZZONE L., PRIETO D.F. Automatic analysis of the difference image for unsupervised change detection. IEEE Transactions on Geoscience and Remote Sensing. 2000, 38, pp. 1171-1182, doi: 10.1109/36.843009.

[3] CELIK T. Change Detection in Satellite Images Using a Genetic Algorithm Approach. IEEE Geoscience and Remote Sensing Letters. 2010, 7, pp. 386-390, doi: 10.1109/LGRS. 2009. 2037024.

[4] CELIK T. Multiscale Change Detection in Multitemporal Satellite Images. IEEE Geoscience and Remote Sensing Letters. 2009, 6, pp. 820-824, doi: 10.1109/LGRS.2009.2026188.

[5] CELIK T. Unsupervised Change Detection in Satellite Images Using Principal Component Analysis and k-Means Clustering. IEEE Geoscience and Remote Sensing Letters. 2009, 6, pp. 772-776, doi: 10.1109/LGRS. 2009.2025059.

[6] CHEN J., CHEN X., CUI X., CHEN J. Change Vector Analysis in Posterior Probability Space: A New Method for Land Cover Change Detection. IEEE Geoscience and Remote Sensing Letters. 2011, 8, pp. 317-321, doi: 10.1109/LGRS.2010.2068537.

[7] CIHLAR J., PULTZ T.J., GRAY A.L. Change detection with synthetic aperture radar. International Journal of Remote Sensing. 1992, 13, pp. 401-414, doi: 10.1080/ 01431169208904045 .

[8] CIVICIOGLU P. Transforming geocentric cartesian coordinates to geodetic coordinates by using differential search algorithm. Computers \& Geosciences. 2012, 46, pp. 229-247, doi: 10. 1016/j . cageo.2011.12.011.

[9] GHOSH A., MISHRA N.S., GHOSH S. Fuzzy clustering algorithms for unsupervised change detection in remote sensing images. Information Sciences. 2011, 181, pp. 699-715, doi: 10. 1016/j.ins. 2010.10.016.

[10] GHOSH S., BRUZZONE L., PATRA S., BOVOLO F., GHOSH A. A Context-Sensitive Technique for Unsupervised Change Detection Based on Hopfield-Type Neural Networks. IEEE Transactions on Geoscience and Remote Sensing. 2007, 45, pp. 778-789, doi: 10. 1109/TGRS. 2006.888861.

[11] GONG M., ZHOU Z., MA J. Change Detection in Synthetic Aperture Radar Images based on Image Fusion and Fuzzy Clustering. IEEE Transactions on Image Processing. 2012, 21 , pp. 2141-2151, doi: 10.1109/TIP.2011.2170702.

[12] GOPAL S., WOODCOCK C. Remote sensing of forest change using artificial neural networks. IEEE Transactions on Geoscience and Remote Sensing. 1996, 34, pp. 398-404, doi: $10.1109 / 36.485117$.

[13] HAO M., ZHANG H., SHI W., DENG K. Unsupervised change detection using fuzzy c-means and MRF from remotely sensed images. Remote Sensing Letters. 2013, 4, pp. 1185-1194, doi: $10.1080 / 2150704 X .2013 .858841$.

[14] HUANG X., FRIEDL M. A. Distance metric-based forest cover change detection using MODIS time series. International Journal of Applied Earth Observation and Geoinformation. 2014, 29, pp. 78-92, doi: 10.1016/j.jag.2014.01.004.

[15] KARABOGA D., OZTURK C. A novel clustering approach: Artificial Bee Colony (ABC) algorithm. Applied Soft Computing. 2011, 11, pp. 652-657, doi: 10.1016/j.asoc.2009.12. 025.

[16] MA W., JIAO L., GONG M., LI C. Image change detection based on an improved rough fuzzy c-means clustering algorithm. International Journal of Machine Learning and Cybernetics. 2013, 5, pp. 369-377, doi: 10.1007/s13042-013-0174-4.

[17] MISHRA N.S., GHOSH S., GHOSH A. Fuzzy clustering algorithms incorporating local information for change detection in remotely sensed images. Applied Soft Computing. 2012, 12, pp. 2683-2692, doi: http://dx.doi.org/10.1016/j.asoc.2012.03.060. 


\section{Neural Network World 2/2016, 141-154}

[18] PACIFICI F., FRATE F.D., SOLIMINI C., EMERY W.J. An Innovative Neural-Net Method to Detect Temporal Changes in High-Resolution Optical Satellite Imagery. Ieee Transactions on Geoscience and Remote Sensing. 2007, 45, pp. 2940-2952, doi: 10.1109/TGRS. 2007.902824.

[19] PATRA S., GHOSH S., GHOSH A. Unsupervised Change Detection in Remote-Sensing Images Using Modified Self-Organizing Feature Map Neural Network. In: Proceedings of the International Conference on Computing: Theory and Applications, (ICCTA '07), Kolkata, India. IEEE, 2007, pp. 716-720, doi: 10.1109/ICCTA.2007.128.

[20] SCHROEDER T.A., WULDER M.A., HEALEY S.P., MOISEN G.G. Detecting post-fire salvage logging from Landsat change maps and national fire survey data. Remote Sensing of Environment. 2012, 122, pp. 166-174, doi: 10.1016/j.rse.2011.10.031.

[21] SUBUDHI B.N., BOVOLO F., GHOSH A., BRUZZONE L. Spatio-contextual fuzzy clustering with Markov random field model for change detection in remotely sensed images. Optics E Laser Technology. 2014, 57, pp. 284-292, doi: 10.1016/j.optlastec.2013.10.003.

[22] TRIANNI V., TUCI E., PASSINO K.M., MARSHALL J.A.R. Swarm Cognition: an interdisciplinary approach to the study of self-organising biological collectives. Swarm Intelligence. 2011, 5, pp. 3-18, doi: 10.1007/s11721-010-0050-8.

[23] VALLE S., LI W., QIN S.J. Selection of the Number of Principal Components: The Variance of the Reconstruction Error Criterion with a Comparison to Other Methods. Industrial \& Engineering Chemistry Research. 1999, 38, pp. 4389-4401, doi: 10.1021/ie990110i.

[24] YA-QIU J. Change detection of enhanced, no-changed and reduced scattering in multitemporal ERS-2 SARimages using the two-thresholds EM and MRF algorithms. In: Proceedings of the 2005 IEEE International Geoscience and Remote Sensing Symposium (IGARSS '05). IEEE, 2005, 6, pp. 3994-3997, doi: 10.1109/IGARSS.2005.1525789.

[25] ZOU W., ZHU Y., CHEN H., SUI X. A Clustering Approach Using Cooperative Artificial Bee Colony Algorithm. Discrete Dynamics in Nature and Society. 2010, 2010, pp. 16, doi: 10. $1155 / 2010 / 459796$. 